\title{
Determinação de fração volumétrica de ferrita em aços inoxidáveis duplex através do Trainable Weka Segmentation
}

\author{
Barbieri, R. $\mathrm{C}^{1}$; Chagas, J. V. $\mathrm{S}^{1}$; Nunes, L. G. S. ${ }^{1 *}$ \\ 1 Programa de Pós-Graduação em Engenharia Mecânica, Universidade Federal do Espírito Santo, Vitória, ES, Brasil. \\ E-mail: lumenag@yahoo.com.br;
}

\begin{abstract}
Resumo
$\mathrm{Na}$ engenharia de materiais, busca-se cada vez mais entender a correlação entre a microestrutura dos materiais e suas propriedades físicas. Com o progresso das técnicas de microscopia e o acoplamento de técnicas de análise de imagens, surgem novos desafios, como realizar uma análise quantitativa e automatizada de imagens microestruturais. Para tanto, deve-se definir uma técnica precisa e eficiente para a segmentação automatizada da imagem. Os métodos de aprendizagem de máquina surgiram como ferramentas poderosas para incluir o conhecimento do operador no processo de segmentação e melhorar a precisão das regiões de interesse. Para segmentar os dados da imagem de entrada, o programa realiza a classificação dos pixels de forma com que cada um pertença exclusivamente a uma classe específica. Uma vez que o classificador é treinado, ele pode ser usado para classificar o restante dos pixels de entrada ou dados de imagem completamente novos. Neste trabalho utilizamos a ferramenta, disponível no software de código aberto Fiji, Trainable Weka Segmentation (TWS) como método de quantificação de fração volumétrica de ferrita em aço duplex. Neste caso, utilizamos sete diferentes micrografias. Visando comprovar a eficiência do TWS na quantificação das fases, os dados foram comparados com medidas realizadas nas mesmas amostras por meio de ferritoscópio, obtendo uma diferença média de aproximadamente $7,7 \%$, diferença justificada devido à baixa quantidade de imagens utilizadas no treinamento. $O$ resultado obtido serve como comprovação de que o TWS pode ser utilizado efetivamente para quantificação de fases em aços duplex.
\end{abstract}

\begin{abstract}
In materials engineering, it is increasingly sought to understand the correlation between the microstructure of materials and their physical properties. With the progress of microscopy techniques and the coupling of image analysis techniques, new challenges arise, such as performing a quantitative and automated analysis of microstructural images. For this purpose, a precise and efficient technique must be defined for the automated segmentation of the image. Machine learning methods have emerged as powerful tools for including operator knowledge in the segmentation process and improving the exactitude of regions of interest. To segment incoming image data, the program turns the targeting problem into a pixel classification problem in which each pixel can be classified as belonging to a specific class. Once the classifier is trained, it can be used to sort the rest of the input pixels or to classify a completely new image data. In this work, we use the tool Trainable Weka Segmentation (TWS), available in open source software Fiji, as a method of quantifying volumetric fraction of ferrite in duplex steel. For this purpose, we use seven different micrographs. Aiming to verify the efficiency of the TWS in the quantification of the phases, we compared the data obtained with measurements made through a ferrite content meter device, obtaining an average difference of $7,7 \%$, difference justified due to the low quantity of images used in the training. The obtained result serves as proof that the TWS can be effectively used for phase quantification in duplex steels.
\end{abstract}

Keywords (Palavras chaves): Fiji, Trainable Weka Segmentation, segmentação de imagens, aços duplex. 


\section{Introdução}

Com a crescente necessidade de extrair dados de grandes quantidades de imagens, torna-se necessária a utilização de ferramentas automatizadas de análise [1]. Um dos principais desafios na extração de dados de uma imagem é sua adequada segmentação, processo no qual se divide uma imagem em várias partes ou segmentos homogêneos. Métodos tradicionais de segmentação são baseados na relação espacial entre os pixels e em suas respectivas intensidades [2].

A segmentação manual, realizada através de inspeção visual, leva em conta a capacidade de discernimento visual humano, que torna o processo mais impreciso e enfadonho. Visando aproveitar também esse conhecimento, recentemente a comunidade científica vem utilizando o aprendizado de máquina para aprimorar a qualidade segmentação de imagens e automatizá-la. Poucos softwares disponibilizam o aprendizado de máquina em conjunto do software de processamento de imagens. Alguns softwares comerciais apresentam essa combinação de ferramentas, porém a utilização desses não é recomendada para fins científicos, pois não é possível ter acesso aos detalhes dos algoritmos. Dentre os softwares livres disponíveis, destaca-se o Trainable Weka Segmentation (TWS) [3]. O TWS é um plugin contido no Fiji, conhecido software de processamento de imagens e é embasado no Waikato Environment for Knowledge Analysis (WEKA), um kit de ferramentas de mineração de dados e aprendizado de máquinas [4]. O TWS tem aplicação em diversos campos da pesquisa científica e já é utilizado extensivamente na área biológica [5]. Dentro da área de engenharia de materiais, o TWS pode ser usado para determinação de fração volumétrica de materiais multifásicos, para contabilização de porosidades, para quantificação de tamanho de contornos de grão entre outros, porém não é usualmente utilizado, sendo escassos os trabalhos encontrados sobre esse tema.

A determinação precisa de fração volumétrica de ferrita em aços inoxidáveis duplex é um fator extremamente importante, pois o desequilíbrio entre a fração volumétrica de ferrita e austenita é um indicativo da precipitação de fases deletérias no material [6]. O objetivo deste trabalho é apresentar o plugin TWS e mostrar que é possível determinar a fração volumétrica de ferrita em aços inoxidáveis duplex através do mesmo com precisão aceitável. Para isso, realizamos as medições em sete amostras do aço AISI UNS 532750 e comparamos com valores obtidos através de medições feitas através de um ferritoscópio.

\section{Metodologia}

A extração de dados através do processamento de imagens consiste em quatro processos: obtenção da imagem, pré-processamento, segmentação e pós-processamento. Um algorítimo simplificado para a segmentação de imagens digitais usando o TWS e a extração de dados é apresentado na tabela 1.

Tabela 1: Etapas para segmentar e extrair dados em processamento de imagens por meio do Fiji e TWS.

\begin{tabular}{|c|l|}
\hline \multicolumn{2}{|c|}{ Etapas } \\
\hline 1 & Abrir o Fiji e carregar a imagem (.tiff) \\
\hline 2 & Carregar o plugin TWS \\
\hline 3 & $\begin{array}{l}\text { Criar e nomear classes suficientes para a } \\
\text { segmentação }\end{array}$ \\
\hline 4 & $\begin{array}{l}\text { Selecionar regiões de interesse e } \\
\text { adicioná-las como amostras das classes }\end{array}$ \\
\hline 5 & $\begin{array}{l}\text { Escolher filtros e parâmetros adequados } \\
\text { para cada tipo de imagem }\end{array}$ \\
\hline 7 & $\begin{array}{l}\text { Treinar o classificador e verificar a } \\
\text { qualidade da segmentação visualmente e } \\
\text { pelo "out of bag error" }\end{array}$ \\
\hline 7 & $\begin{array}{l}\text { Repetir passo 4-6 até um resultado } \\
\text { satisfatório }\end{array}$ \\
\hline 8 & Gerar e salvar a imagem segmentada \\
\hline 9 & $\begin{array}{l}\text { Salvar o classificador treinado e os dados } \\
\text { para aplicar em futuras imagens }\end{array}$ \\
\hline 10 & $\begin{array}{l}\text { Selecionar a medição de área em "Set } \\
\text { measurements" }\end{array}$ \\
\hline 11 & $\begin{array}{l}\text { Usar o "Particle analyzer" na imagem } \\
\text { segmentada e binarizada }\end{array}$ \\
\hline
\end{tabular}

\subsection{Obtenção das imagens}

Imagens de alta qualidade e com contraste entre as fases a serem quantificadas são fundamentais para a aquisição de dados precisos. A obtenção de imagens foi inteiramente realizada no Laboratório de Tribologia e Corrosão de Materiais (TRICORRMAT) da UFES. Sete amostras do aço inoxidável duplex AISI UNS S32750 foram preparadas por técnicas metalográficas usuais. Com o objetivo de revelar a microestrutura ferrítica realizamos um ataque eletrolítico com uma solução de hidróxido de potássio $(\mathrm{KOH})$ por 
$7 \mathrm{~s}$ sob uma tensão de $2,5 \mathrm{~V}$. As micrografias foram obtidas por meio do microscópio Nikon Eclipse MA200, com ampliações de 500x e 1000x.

\subsection{Pré-processamento}

O pré-processamento é o conjunto de operações feitas sobre a imagem com o objetivo de facilitar e aumentar a precisão na segmentação. Vale destacar que as operações do pré-processamento diferem de imagem para imagem. A escolha das operações deve ser feita com base na condição da imagem e em quais informações se deseja extrair da mesma. No presente trabalho, o pré-processamento consistiu de duas etapas: subtração de background e corte. A subtração de background é uma operação realizada sobre a imagem com o objetivo de minimizar a diferença de iluminação em sua extensão. As imagens também foram cortadas para descartar regiões com texto, como escala, e aquelas regiões que continham diferenças acentuadas de iluminação. Essas diferenças prejudicam o bom funcionamento do TWS. As operações de corte e subtração de background foram realizadas no Fiji.

\subsection{Segmentação}

O TWS tem como algoritmo padrão para classificação, o método Random Forest, amplamente difundido no campo da biomédica. Essa técnica de aprendizado de máquina é reconhecida como uma das melhores técnicas de classificação devido a sua baixa sensibilidade ao desbalanceamento na quantidade de dados de classes distintas e a sua capacidade de lidar com tarefas nãolineares [7]. O Random Forest consiste em duas etapas: treinamento e teste. A seleção das classes é feita pelo operador. O operador mostra para o software qual pixel pertence a cada classe (treinamento) e o software se encarrega de aplicar essa classificação ao longo de toda imagem, segmentando-a (teste). Cada pixel pertence a apenas uma fase (austenita ou ferrita) o que evidencia a importância de imagem com bom contraste entre as fases. Se o teste não estiver satisfatório, é possível treinar o classificador várias vezes até que o teste produza resultados aceitáveis.

No TWS é necessário que seja feita a seleção de filtros a serem utilizados no treinamento de acordo com a necessidade do operador. Existem filtros detectores de bordas, filtros de textura, redutores de ruído e detectores de membrana [1]. Deve-se começar o treinamento usando poucos filtros. Durante o teste, é possível visualizar na janela de log do TWS se um filtro aumentou ou reduziu o erro na classificação. A avaliação da qualidade do filtro é feita com base no aumento ou redução do erro: mantêm-se os filtros que reduzem o erro e removem-se os filtros que aumentam. Neste trabalho foram selecionados os filtros "anisotropic diffusion" e "structure" em todas as imagens. Dentro dos filtros é possível variar outros parâmetros de maneira a aperfeiçoar a segmentação. Uma vez finalizado o treinamento, é possível salvar o classificador para aplicá-lo a imagens similares. O TWS também permite o uso do classificador em várias imagens de uma só vez, embora esse processo aumente a demanda de processamento computacional.

\subsection{Pós-processamento}

A imagem segmentada deve ser binarizada para que 0 Fiji consiga extrair seus dados. $O$ processo de binarização faz com que a imagem assuma cor preta ou branca (Figura 1) de acordo com a segmentação realizada no TWS. A ferramenta "Particle Analyzer" do Fiji extrai dados como fração de área e perímetro de cada fase. Dentro desta ferramenta, é possível selecionar o tamanho mínimo de partícula a ser levada em consideração e preencher pequenas descontinuidades nas fases (fill holes). O tamanho mínimo de partícula escolhido foi de cinco pixels e a opção de preencher descontinuidades foi selecionada. Os valores de fração entre as áreas de cada classe foram então extraídos.
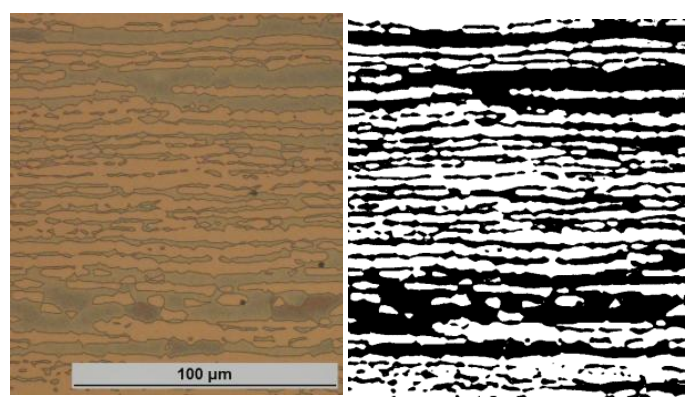

Figura 1: Comparação entre a microscopia original da amostra S7 e o resultado da segmentação realizado pelo TWS.

\section{Discussão dos resultados}

A Tabela 2 apresenta os resultados percentuais do teor de ferrita presente em cada amostra, calculados através do ferritoscópio e da análise 
de partículas da imagem segmentada obtida pelo TWS. Calculou-se também a diferença percentual entre os métodos.

Tabela 2: Teor de ferrita presente em cada amostra obtida através de ferritoscópio e análise de imagens (TWS)

\begin{tabular}{|c|c|c|c|}
\hline Amostras & $\begin{array}{c}\text { Ferritoscópio } \\
(\%)\end{array}$ & $\begin{array}{c}\text { TWS } \\
(\%)\end{array}$ & $\begin{array}{c}\text { Diferença } \\
\text { entre } \\
\text { métodos (\%) }\end{array}$ \\
\hline S1 & 54,99 & 51,83 & 5,7 \\
\hline S2 & 56,91 & 50,52 & 11,2 \\
\hline S3 & 52,4 & 48,35 & 7,7 \\
\hline S4 & 52,57 & 53,03 & 0,9 \\
\hline S5 & 51,67 & 44,48 & 13,9 \\
\hline S6 & 50,73 & 48,84 & 3,7 \\
\hline S7 & 45,73 & 53,16 & 16,2 \\
\hline
\end{tabular}

Analisando os resultados dos dois métodos de quantificação de fases, verifica-se que há uma diferença máxima entre os métodos de $16,2 \%$ com uma média de $7,7 \%$. Isto ocorre na medida em que a análise da imagem foi pontual, se limitando a uma área microscópica da peça, enquanto a análise do ferritoscópio mede a fração ferromagnética de uma região macroscópica, tendo uma análise mais global e com maior relevância estatística. Esse erro poderia ser minimizado através do aumento do número de imagens micrográficas a ser analisada em cada amostra, o que contribuiria para uma melhor eficiência no treinamento. Nota-se também nas amostras S4 e S6 a diferença entre os métodos foi menor, isso se deve a elevada qualidade na obtenção da imagem e contraste entre as fases dessas microscopias, o que permitiu uma melhor segmentação e quantificação das mesmas pelo programa.

\section{Conclusão}

O Trainable Weka Segmentation se mostrou uma ferramenta de análise de imagens eficaz para ser utilizada na quantificação de fases em aços duplex. Comparando com um método quantitativo, a análise de imagens apresentou em média uma diferença percentual entre os métodos de $7,7 \%$. É possível reduzir essa diferença ao segmentar mais imagens para cada amostra, melhorando a estatística do processo.

Através desta ferramenta mostrou-se ser possível quantificar o percentual de fases em aço duplex somente através de análise de imagens microestruturais, automatizando o processo.

\section{Agradecimentos}

Os autores agradecem a CAPES e FAPES pelo apoio financeiro, ao TRICORRMAT da UFES pela utilização do microscópio ótico para obtenção das imagens e a PETROBRAS pela cessão do material.

\section{Referências}

[1] ARGANDA-CARRERAS, I. et. al. Bioinformatics, v. 33, p. 2424-2426, 2017.

[2] ZAITOUN, N. M.; AQEL, M. J., Procedia Computer Science, v. 65, p. 797-806, 2015.

[3] SCHINDELIN, J. et. al. Nature Methods, v. 9, p. 676-682, 2012.

[4] HALI, M. et. al. SIGKDD Explor. Newsl. v. 11. p. 10-18, 2008.

[5] AFFONSO, C. et. al, Expert Systems with Applications, v. 85, p. 114-122, 2017

[6] AZEVEDO, C. R. F. et. al., Engineering Failure Analysis, v. 97, p. 161-188, 2019.

[7] KOCH, P.; LUND, C.; ROSENKRANZ, J. Minerals Engineering, v. 136, p. 99-109, 2019. 